\title{
QUADRATIC SPHERICITY TEST FOR BLIND DETECTION OVER TIME-VARYING FREQUENCY-SELECTIVE FADING CHANNELS
}

\author{
Josep Font-Segura, Jaume Riba, Javier Villares and Gregori Vazquez
}

\author{
Signal Theory and Communications Department, Technical University of Catalonia (UPC) \\ D5-\{214, 116, 115, 204\}, Campus Nord UPC, Jordi Girona 1-3, 08034 Barcelona, Spain \\ Email: \{josep.font-segura, jaume.riba, javier.villares, gregori.vazquez\}@upc.edu
}

\begin{abstract}
This paper addresses the problem of blind detection of a wide-sense stationary (WSS) signal over fading channels. We propose a test statistic which is optimal from a correlationmatching perspective that shows invariance with respect to the noise power and the channel gain. In the blind scenario, we derive the quadratic sphericity test (QST) which exploits the structure of the fading channel as a squared mean to arithmetic mean ratio of the eigenvalues of the autocorrelation matrix of the observations. We provide numerical results to assess the performance of the QST in several fading scenarios, as well as the benchmarking to other blind and non-blind detectors.
\end{abstract}

Index Terms - Statistical signal detection, spectrum sensing, blind, fading, cognitive radio.

\section{INTRODUCTION}

Cognitive radio holds a big promise as the future technology capable of overcoming the current scarcity of spectrum resources in wireless communications [1]. The coexistence between primary or licensed users and secondary or unlicensed users involves a wide range of signal processing problems, mostly in the interweave paradigm [2] in which secondary users opportunistically transmit only in the available unused licensed resources. Hence, signal detection or spectrum sensing is an important task in interweave cognitive radio [3], as interference will occur in an event of a false spectral hole detection. Optimal signal detection in fading channels would exploit the fading coefficients of the channel. However, this knowledge is either impractical in decentralized cognitive radio networks, or it contains errors if estimated from primary pilots [4].

In this paper, we derive a blind detector based on the squared mean to arithmetic mean ratio of the eigenvalues

This work has been partially funded by the Spanish Government under TEC2010-21245-C02-01 (DYNACS), CONSOLIDER INGENIO CSD200800010 (COMONSENS), CENIT CEN-20101019 (THOFU), and the Catalan Government (AGAUR) under Grant 2009SGR1236 and Fellowship FI-2010. of the autocorrelation matrix of the observations, which we denote as the quadratic sphericity test (QST). We first derive a test statistic which is optimal in terms of a correlationmatching of the second-order statistics of the problem, and which shows invariance with respect to the noise power and the channel gain. We then derive the QST by optimizing the correlation-matching cost function with respect to the unknown channel fading coefficients.

Prior work related to the QST is detailed as follows. Spectrum sensing detectors based on the autocorrelation matrix of the observations are reported in [5-7]. These works exploit the structure of the correlation matrix of the transmitted legacy signal, but fading is not considered. Spectrum sensing detectors based on the eigenvalues of the sample covariance matrix include the maximum to minimum eigenvalue (MME) [8] and the normalized maximum eigenvalue (NME) [9]. We consider the latter in the numerical results for comparison. There is also a large body of spectrum sensing detectors taking into account unknown parameters, e.g., [10-14]. However, these works exploit the spatial correlation that arises from the use of multiple antennas. In this work we rather consider a single antenna receiver in time-varying frequencyselective channels. Finally, the works $[15,16]$ consider signal detection in fading channels, but considering prior knowledge on the statistics of the channel.

The rest of the paper is organized as follows. The signal detection problem in time-varying frequency-selective channels is formulated in Sec. 2. The QST is formulated and proved in Sec. 3. Sec. 4 illustrates the numerical results, and Sec. 5 concludes the paper.

\section{PROBLEM FORMULATION}

The detection of a primary wide-sense stationary (WSS) signal is cast as the following binary hypotheses testing problem:

$$
\begin{aligned}
& \mathcal{H}_{0}: \mathbf{y}=\sqrt{P_{n}} \mathbf{w}, \\
& \mathcal{H}_{1}: \mathbf{y}=\sqrt{\operatorname{SNR} P_{n}} \mathbf{x}+\sqrt{P_{n}} \mathbf{w}
\end{aligned}
$$

where $\mathbf{y}=[y(0), \ldots, y(N-1)]^{T}$ collects $N$ consecutive samples as observations, $\mathbf{w}$ denotes the unit power noise sam-

(C) 2013 IEEE. Personal use of this material is permitted. Permission from IEEE must be obtained for all other uses, in any current or future media, including reprinting/republishing this material for advertising or promotional purposes, creating new collective works, for resale or redistribution to servers or lists, or reuse of any copyrighted component of this work in other works. 
ples, $\mathbf{x}$ denotes the unit power signal samples, $P_{n}$ denotes the noise power and SNR denotes the signal-to-noise ratio.

In this work, the signal $\mathrm{x}$ is the output of a time-varying linear system (LTV) given as ${ }^{1}$

$$
\mathbf{x}=\mathbf{A}_{\mathbf{H}} \mathbf{s}
$$

where $\mathbf{s}$ is a column vector containing $K$ digitally modulated symbols, and $\mathbf{A}_{\mathbf{H}}$ models the time-varying frequencyselective nature of the fading channel as ${ }^{2}$

$$
\mathbf{A}_{\mathbf{H}}=\left[\begin{array}{cccc}
h_{0}(1) & 0 & \cdots & 0 \\
\vdots & h_{1}(1) & & \vdots \\
h_{0}(L) & \vdots & \ddots & 0 \\
0 & h_{1}(L) & & h_{K-1}(1) \\
\vdots & & \ddots & \vdots \\
0 & \cdots & 0 & h_{K-1}(L)
\end{array}\right]
$$

For sake of notation we define the channel matrix as

$$
\mathbf{H}=\left[\mathbf{h}_{0}, \ldots, \mathbf{h}_{K-1}\right]
$$

where each column contains the channel impulse response at time index $k$, i.e.,

$$
\mathbf{h}_{k}=\left[h_{k}(1), \ldots, h_{k}(L)\right]^{T} .
$$

\section{BLIND DETECTOR FOR TIME-VARYING FREQUENCY-SELECTIVE CHANNELS}

In this work, we adopt the following test statistic which shows invariance with respect to the time varying frequencyselective channel gain and the noise variance, given by

$$
T(\mathbf{y})=\frac{1}{\mathbf{y}^{H} \mathbf{y}} \max _{\mathbf{H}} \frac{\operatorname{tr}\left(\mathbf{H} \mathbf{H}^{H} \hat{\mathbf{R}}\right)}{\left\|\mathbf{H} \mathbf{H}^{H}\right\|_{F}} \geq \gamma,
$$

where

$$
\hat{\mathbf{R}}=\frac{1}{K} \sum_{k=0}^{K-1} \mathbf{y}_{k} \mathbf{y}_{k}^{H}
$$

is the $L \times L$ autocorrelation matrix of the observations, $\gamma$ is the detection threshold, and

$$
\mathbf{y}_{k}=[y(k), \ldots, y(k+L-1)]^{T} .
$$

We refer to Sec. 3.1 for the correlation-matching proof of (6). In general, the solution to the maximization problem (6) leads

\footnotetext{
${ }^{1}$ For simplicity we assume that the convolution matrix $\mathbf{A}_{\mathbf{H}}$ models the pulse shaping at transmission, the fading nature of the channel, and the matched-filtering at the receiver.

${ }^{2}$ The model adopted in (3) illustrates how each of the transmitted symbol $s(k)$ is propagated through the channel with fading coefficients $\mathbf{h}_{k}$.
}

to the following blind detector as a function of the eigenvalues of the autocorrelation matrix of the observations ${ }^{3}$

$$
T_{\mathrm{QST}}(\mathbf{y}) \approx \frac{\sqrt{\sum_{i=1}^{L} \lambda_{i}^{2}(\hat{\mathbf{R}})}}{\sum_{i=1}^{L} \lambda_{i}(\hat{\mathbf{R}})}
$$

The proof of (9) is developed in Sec. 3.2. We denote this detector as the quadratic sphericity test (QST) as it evaluates the squared mean of the eigenvalues of the autocorrelation matrix of the observations divided by the arithmetic mean of the eigenvalues. Therefore, from the structure of the autocorrelation matrix (7), we observe that the QST is a measure of dispersion of the eigenvalues in the $L$-dimensional space that arises from the structure of the fading channel $\mathbf{H}$. Under $\mathcal{H}_{0}$, the QST will observe a flat distribution of the eigenvalues, whereas under $\mathcal{H}_{1}$ the distribution of the eigenvalues will depend on the rank of $\mathbf{H}$. Finally, because slow-fading scenarios have lower variability, the eigenvalues of $\hat{\mathbf{R}}$ will exhibit large dispersion as some of them will approach zero, whereas in fast-fading scenarios the dispersion will be lower.

\subsection{Proof of the Invariant Test Statistic (6)}

We proof the optimality in the correlation-matching sense of the blind detector for time-varying frequency-selective channels (6). Specifically, we adopt the negative correlationmatching between the normalized autocorrelation of the data and a scaled version of the desired signal autocorrelation

$$
T(\mathbf{y})=\max _{\substack{\mathbf{R}_{x} \in \Omega \\ 0<\gamma<1}}-\left\|\frac{\mathbf{y} \mathbf{y}^{H}}{\mathbf{y}^{H} \mathbf{y}}-\gamma \mathbf{R}_{x}\right\|_{\eta}^{2},
$$

where $\gamma$ is the coherence factor defined as

$$
\gamma \approx \frac{\mathrm{SNR}}{\mathrm{SNR}+1}
$$

and it conveniently scales the signal autocorrelation matrix $\mathbf{R}_{x}$ of the signal samples (2), i.e.,

$$
\mathbf{R}_{x}=\mathbf{A}_{\mathbf{H}} \mathbf{A}_{\mathbf{H}}^{H} \text {. }
$$

In (10), the maximization of the autocorrelation matrix is done over the space of all Hermitian matrices $\Omega$, and the correlation-matching is implemented through a quadratic norm $\eta$. Expanding the norm in (10) it follows that

$$
-\left\|\frac{\mathbf{y} \mathbf{y}^{H}}{\mathbf{y}^{H} \mathbf{y}}-\gamma \mathbf{R}_{x}\right\|_{\eta}^{2}=-1+2 \gamma \frac{\mathbf{y}^{H} \mathbf{R}_{x} \mathbf{y}}{\mathbf{y}^{H} \mathbf{y}}-\gamma^{2}\left\|\mathbf{R}_{x}\right\|_{\eta}^{2} .
$$

The maximization with respect to the coherence factor $\gamma$ leads to the following expression

$$
\hat{\gamma}=\frac{\mathbf{y}^{H} \mathbf{R}_{x} \mathbf{y}}{\left(\mathbf{y}^{H} \mathbf{y}\right)\left\|\mathbf{R}_{x}\right\|_{\eta}^{2}} .
$$

\footnotetext{
${ }^{3}$ Note that the numerator and denominator of (9) can be computed as the squared root of $\operatorname{tr}\left(\hat{\mathbf{R}}^{2}\right)$ and as $\operatorname{tr}(\hat{\mathbf{R}})$ with reduced complexity, respectively.
} 
By substituting (14) into (13), (10) simplifies to

$$
-\left\|\frac{\mathbf{y} \mathbf{y}^{H}}{\mathbf{y}^{H} \mathbf{y}}-\hat{\gamma} \mathbf{R}_{x}\right\|_{\eta}^{2}=-1+\frac{\left(\mathbf{y}^{H} \mathbf{R}_{x} \mathbf{y}\right)^{2}}{\left(\mathbf{y}^{H} \mathbf{y}\right)^{2}\left\|\mathbf{R}_{x}\right\|_{\eta}^{2}} .
$$

Finally, removing the additive constants and taking the squared root it follows that the maximization problem (10) is equivalent to

$$
T(\mathbf{y})=\frac{1}{\mathbf{y}^{H} \mathbf{y}} \max _{\mathbf{R}_{x} \in \Omega} \frac{\mathbf{y}^{H} \mathbf{R}_{x} \mathbf{y}}{\left\|\mathbf{R}_{x}\right\|_{\eta}} .
$$

To this point, we have derived an optimal test from a correlation-matching perspective general for any received signal model $\mathbf{x}$. For fading channels, the received signal is given by (2), and its autocorrelation matrix as (12). Therefore, the numerator of (16) can be expressed as

$$
\mathbf{y}^{H} \mathbf{R}_{x} \mathbf{y}=\sum_{k=0}^{K-1} \mathbf{h}_{k}^{H} \mathbf{y}_{k} \mathbf{y}_{k}^{H} \mathbf{h}_{k}
$$

where $\mathbf{h}_{k}$ and $\mathbf{y}_{k}$ have been previously defined in (5) and (8). The structure of (17) makes the optimization with respect to the channel coefficients a difficult problem. To simplify it, we resort to large data records. Employing the trace operator and dividing by $K$ we can express (17) as

$$
\operatorname{tr}\left(\frac{1}{K} \sum_{k=0}^{K-1} \mathbf{h}_{k} \mathbf{h}_{k}^{H} \mathbf{y}_{k} \mathbf{y}_{k}^{H}\right)
$$

If $K$ is large, we have that in virtue of the law of large numbers (LLN), or equivalently the ergodicity property, the sample mean approaches to the statistical mean. In addition, as the channel realization are statistically independent on the signal plus noise realizations, the statistical mean can be further factorized. As a result of both assessments,

$$
\begin{aligned}
\operatorname{tr}\left(\frac{1}{K} \sum_{k=0}^{K-1} \mathbf{h}_{k} \mathbf{h}_{k}^{H} \mathbf{y}_{k} \mathbf{y}_{k}^{H}\right) \asymp \\
\quad \operatorname{tr}\left(\frac{1}{K} \sum_{k=1}^{K-1} \mathbf{h}_{k} \mathbf{h}_{k}^{H} \times \frac{1}{K} \sum_{k=1}^{K-1} \mathbf{y}_{k} \mathbf{y}_{k}^{H}\right)
\end{aligned}
$$

Hence, removing constants the numerator yields to

$$
\mathbf{y}^{H} \mathbf{R}_{x} \mathbf{y} \asymp \operatorname{tr}\left(\mathbf{H} \mathbf{H}^{H} \hat{\mathbf{R}}\right),
$$

where $\hat{\mathbf{R}}$ is defined from (19) as in (7). On the other hand, we adopt the denominator norm as

$$
\left\|\mathbf{R}_{x}\right\|_{\eta}=\left\|\mathbf{H H}^{H}\right\|_{F}
$$

where $\|\cdot\|_{F}$ is the Frobenius norm, which preserves the invariance property of the detector. Finally, as both (20) and
(21) strictly depend on the channel matrix $\mathbf{H}$, the maximization with respect to $\mathbf{R}_{x}$ is equivalent to the maximization with respect to $\mathbf{H}$. As a result, the test (16) rewrites

$$
T(\mathbf{y})=\frac{1}{\mathbf{y}^{H} \mathbf{y}} \max _{\mathbf{H}} \frac{\operatorname{tr}\left(\mathbf{H} \mathbf{H}^{H} \hat{\mathbf{R}}\right)}{\left\|\mathbf{H} \mathbf{H}^{H}\right\|_{F}},
$$

as we wanted to show.

\subsection{Proof of the QST (9)}

From the invariant detector (6) we express the Hermitian matrix $\mathbf{H H}^{H}$ as a linear combination of its eigenvalues and eigenvectors

$$
\mathbf{H H}^{H}=\sum_{i=1}^{L} \mu_{i} \cdot \mathbf{v}_{i} \mathbf{v}_{i}^{H},
$$

where $\mu_{i}$ and $\mathbf{v}_{i}$ denote the corresponding eigenvalues and eigenvectors, respectively. The invariant detector then admits the following optimization problem form:

$$
T(\mathbf{y})=\frac{1}{\mathbf{y}^{H} \mathbf{y}} \max _{\left\{\mu_{i}, \mathbf{v}_{i}\right\}} \sum_{i=1}^{L} \mu_{i} \mathbf{v}_{i}^{H} \hat{\mathbf{R}} \mathbf{v}_{i}
$$

subject to $\sum_{i=1}^{L} \mu_{i}^{2}=1$, and $\mathbf{v}_{i}^{H} \mathbf{v}_{j}=\delta_{i j}$ for $i, j=1, \ldots, L$. First, we solve for the eigenvectors from the Lagrangian $L\left(\mathbf{v}_{1}, \ldots, \mathbf{v}_{L}\right)=\sum_{i=1}^{L} \mu_{i} \mathbf{v}_{i}^{H} \hat{\mathbf{R}} \mathbf{v}_{i}+\sum_{i=1}^{L} \rho_{i}\left(1-\mathbf{v}_{i}^{H} \mathbf{v}_{i}\right)$, where $\rho_{1}, \ldots, \rho_{L}$ are the Lagrange multipliers. By computing the derivative and setting it to zero, it yields to $\hat{\mathbf{R}} \mathbf{v}_{i}=\left(\rho_{i} / \mu_{i}\right) \mathbf{v}_{i}$. That is, the eigenvectors of $\mathbf{H} \mathbf{H}^{H}$ are given by

$$
\mathbf{v}_{i}=\mathbf{u}_{i}
$$

where $\mathbf{u}_{i}$ denote the eigenvectors of $\hat{\mathbf{R}}$, and $\rho_{i} / \mu_{i}$ are the eigenvalues of $\hat{\mathbf{R}}$, which we denote by $\lambda_{i}$. Notice that the orthonormality constraint on the vectors $\mathbf{v}_{1}, \ldots, \mathbf{v}_{L}$ is automatically fulfilled as the eigenvectors of $\hat{\mathbf{R}}$ are orthonormal. Next, we solve for the eigenvalues as $L\left(\mu_{1}, \ldots, \mu_{L}\right)=$ $\sum_{i=1}^{L} \mu_{i} \lambda_{i}+\mu\left(1-\sum_{i=1}^{L} \mu_{i}^{2}\right)$. By computing the derivative and setting it to zero, we obtain that

$$
\mu_{i}=\frac{\lambda_{i}}{\sqrt{\sum_{j=1}^{L} \lambda_{j}^{2}}}
$$

Finally, asymptotically for large $K$, we can approximate the $\mathbf{y}^{H} \mathbf{y}$ term by

$$
\mathbf{y}^{H} \mathbf{y} \asymp \frac{N}{L} \operatorname{tr}(\hat{\mathbf{R}})=\frac{N}{L} \sum_{i=1}^{L} \lambda_{i} .
$$

Using (25), (26) and (27) into (24) and removing constants, we obtain (9). 


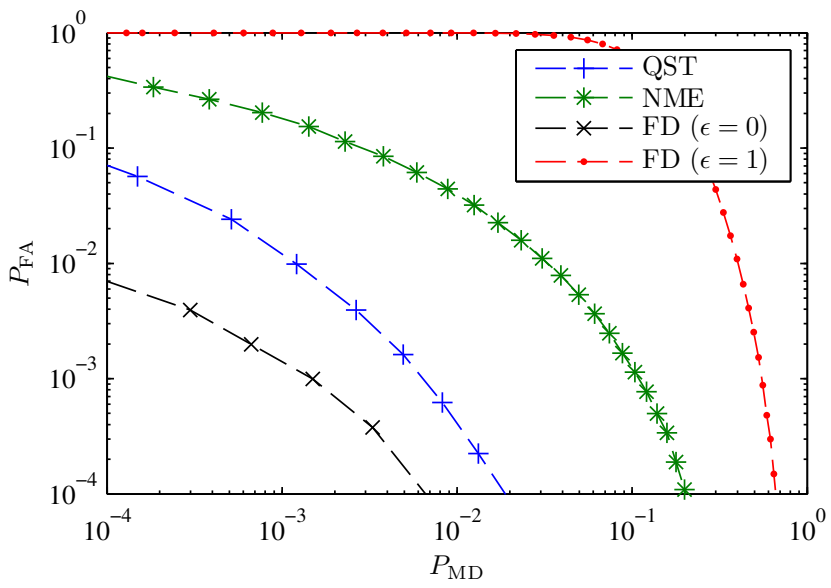

Fig. 1. Receiver operating characteristics (ROC) of the QST (9), the FD (28) with $\epsilon=0$ and $\epsilon=1$, as well as the NME (30) in a slow-fading scenario.

\section{NUMERICAL RESULTS}

In this section, we provide numerical results to assess the performance of the QST (9) in terms of the receiver operating characteristics (ROC) curve (missed-detection probability $P_{\mathrm{MD}}$ versus false-alarm probability $P_{\mathrm{FA}}$ ) in several scenarios. As a benchmarking, we will consider the following additional signal detectors. On the one hand, we consider the feature detector (FD) which is given by the invariant detector (6) with side information of the channel coefficients $\hat{\mathbf{H}}$, i.e.,

$$
T_{\mathrm{FD}}(\mathbf{y})=\frac{1}{\mathbf{y}^{H} \mathbf{y}} \frac{\operatorname{tr}\left(\hat{\mathbf{H}} \hat{\mathbf{H}}^{H} \hat{\mathbf{R}}\right)}{\left\|\hat{\mathbf{H}} \hat{\mathbf{H}}^{H}\right\|_{F}},
$$

with

$$
\hat{\mathbf{H}}=\sqrt{\frac{1}{1+\epsilon^{2}}} \mathbf{H}+\sqrt{\frac{\epsilon^{2}}{1+\epsilon^{2}}} \boldsymbol{\Delta},
$$

where $\mathbf{H}$ is the true channel matrix, and $\boldsymbol{\Delta}$ is uniformly distributed within the range $\|\Delta\| \leq 1$ to model the error incurred in the estimation of the channel in pilot-based cognitive radio networks. We will consider both the perfectly known case $\epsilon=0$, and the FD with channel uncertainty $\epsilon=1$. On the other hand, we consider the NME, given by [9]

$$
T_{\mathrm{NME}}(\mathbf{y}) \approx \frac{\lambda_{1}(\hat{\mathbf{R}})}{\sum_{i=1}^{L} \lambda_{i}(\hat{\mathbf{R}})} .
$$

We simulate the transmission of $K$ QPSK symbols over a fading channel $\mathbf{H}$ containing $K$ realizations of the channel coefficients according to the block fading model [17], i.e., throughout $K$ channel realizations, we assume that the channel response $\mathbf{h}$ remains constant through $T$ realizations and then changes independently to another value. In the sequel, we consider the slow fading scenario $(T \gg K)$, and the fast

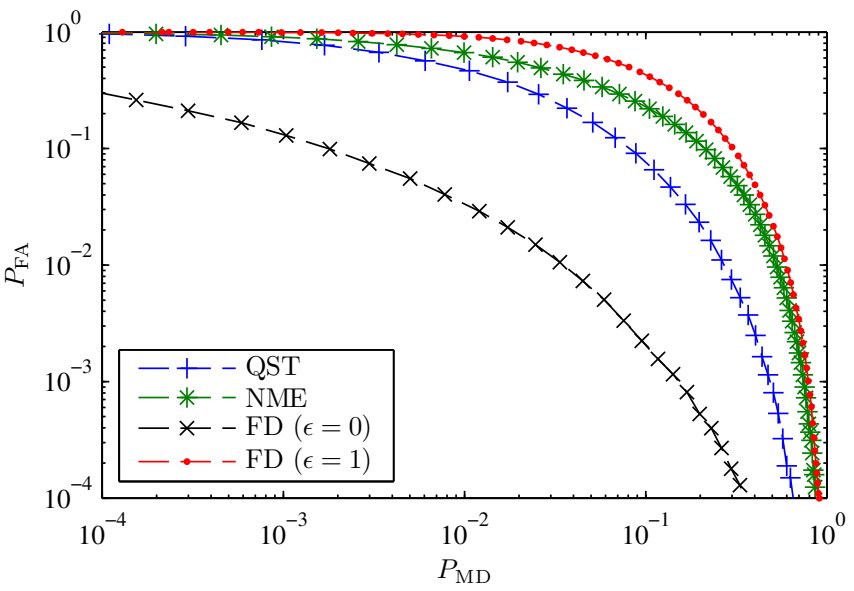

Fig. 2. Receiver operating characteristics (ROC) of the QST (9), the FD (28) with $\epsilon=0$ and $\epsilon=1$, as well as the NME (30) in a fast-fading scenario.

fading scenario $(1 \leq T \ll K)$. The local receiver operates at a SNR of $5 \mathrm{~dB}$ and acquires $N=256$ samples. We consider a channel of length $L=12$ unit variance i.i.d. lags.

The ROC of the QST (9), the FD (28) with $\epsilon=0$ and $\epsilon=1$, as well as the NME (30) in a slow-fading and fastfading $(T=5)$ scenarios are depicted in Fig. 1 and Fig. 2, respectively. As it can be appreciated, the FD with channel errors incurs a severe performance loss compared to the FD without errors in both scenarios due to the mismatching between the true channel and the available side information. On the other hand, both QST and NME outperform the FD with errors. In general, we observe that the QST has a performance gain with respect to the NME, as the NME is only concerned in extracting a channel feature corresponding to the largest eigenvalue. Finally, whereas detecting the channel structure in fast-fading scenarios is a challenging task for both QST and NME, in the slow-fading scenario the QST provides good performance characteristics and approaches the upper-bound established by the FD with perfectly known channel.

\section{CONCLUSIONS}

In this paper, we have introduced the quadratic sphericity test (QST) for blind detection of wide-sense stationary (WSS) signals in time-varying frequency-selective fading channels. We have derived a correlation-matching optimal invariant test statistic, and we have shown that the QST is the solution in the blind case. The QST evaluates the ratio between the squared mean and the arithmetic mean of the eigenvalues of the autocorrelation matrix. We have provided numerical results to assess and benchmark the performance of the QST in slow and fast-fading scenarios. 


\section{REFERENCES}

[1] J. Mitola III and G. Q. Maguire, "Cognitive radio: making software radios more personal," IEEE Personal Commun. Mag., vol. 6, no. 4, pp. 13-18, Aug. 1999.

[2] A. Goldsmith, S. A. Jafar, I. Maric, and S. Srinivasa, "Breaking spectrum gridlock with cognitive radios: An information theoretic perspective," Proc. IEEE, vol. 97, no. 5, pp. 894-914, May 2009.

[3] S. Haykin, D. J. Thomson, and J. H. Reed, "Spectrum sensing for cognitive radio," Proc. IEEE, vol. 97, no. 5, pp. 849-877, May 2009.

[4] A. Ghasemi and E.S. Sousa, "Spectrum sensing in cognitive radio networks: requirements, challenges and design trade-offs," IEEE Commun. Mag., vol. 46, no. 4, pp. 32-39, Apr. 2008.

[5] A. I. Perez-Neira, M. A. Lagunas, M. A. Rojas, and P. Stoica, "Correlation matching approach for spectrum sensing in open spectrum communications," IEEE Trans. Signal Process., vol. 57, no. 12, pp. 4823-4836, Dec. 2009.

[6] Y. Zeng and Y.-C. Liang, "Spectrum-sensing algorithms for cognitive radio based on statistical covariances," IEEE Trans. Veh. Technol., vol. 58, no. 4, pp. 1804-1815, May 2009.

[7] Z. Quan, W. Zhang, S. J. Shellhammer, and A. H. Sayed, "Optimal spectral feature detection for spectrum sensing at very low snr," IEEE Trans. Commun., vol. 59, no. 1, pp. 201-212, Jan. 2011.

[8] Yonghong Zeng and Ying chang Liang, "Eigenvaluebased spectrum sensing algorithms for cognitive radio," IEEE Trans. Commun., vol. 57, no. 6, pp. 1784-1793, June 2009.

[9] Yonghong Zeng, Ying Chang Liang, and Rui Zhang, "Blindly combined energy detection for spectrum sensing in cognitive radio," IEEE Signal Process. Lett., pp. 649-652, 2008.

[10] R. Zhang, T. J. Lim, Y-C. Liang, and Y. Zeng, "Multiantenna based spectrum sensing for cognitve radios: a GLRT approach," IEEE Trans. Commun., vol. 58, no. 1, pp. 84-88, Jan. 2010.

[11] A. Taherpour, M. Nasiri-Kenari, and S. Gazor, "Multiple antenna spectrum sensing in cognitive radios," IEEE Trans. Wireless Commun., vol. 9, no. 2, pp. 814-823, Feb. 2010.

[12] D. Ramirez, G. Vazquez-Vilar, R. Lopez-Valcarce, J. Via, and I. Santamaria, "Detection of rank- $P$ signals in cognitive radio networks with uncalibrated multiple antennas," IEEE Trans. Signal Process., vol. 59, no. 8, pp. 3764-3774, Aug. 2011.

[13] G. Vazquez-Vilar, R. Lopez-Valcarce, and J. Sala, "Multiantenna sectrum sensing exploiting spectral a priori information," IEEE Trans. Wireless Commun., vol. 10, no. 12, pp. 4345-4355, Dec. 2011.

[14] J. Sala-Alvarez, G. Vazquez-Vilar, and R. LopezValcarce, "Multiantenna GLR detection of rank-one signals with known power spectrum in white noise with unknown spatial correlation," IEEE Trans. Signal Process., vol. 60, no. 6, pp. 3065-3078, June 2012.

[15] M. Mohammadkarimi, B. Mahboobi, and M. Ardebilipour, "Optimal spectrum sensing in fast fading Rayleigh channel for cognitive radio," IEEE Commun. Lett., vol. 15, no. 10, pp. 1032-1034, Oct. 2011.

[16] J. Font-Segura and X. Wang, "GLRT-based spectrum sensing for cognitive radio with prior information," IEEE Trans. Commun., vol. 58, no. 7, pp. 2137-2146, July 2010.

[17] E. Biglieri, J. Proakis, and S. Shamai, "Fading channels: information-theoretic and communications aspects," IEEE Trans. Inf. Theory, vol. 44, no. 6, pp. 2619 2692, Oct. 1998. 